\title{
Mathematical Modeling and a Hybrid NSGA-II Algorithm for Process Planning Problem Considering Machining Cost and Carbon Emission
}

\author{
Jin Huang ${ }^{1,2}$, Liangliang Jin ${ }^{3, *}$ and Chaoyong Zhang ${ }^{1}$ \\ 1 School of Mechanical Science \& Engineering, Huazhong University of Science \& Technology, \\ Wuhan 430074, China; d201077074@hust.edu.cn (J.H.); zcyhust@hust.edu.cn (C.Z.) \\ 2 School of Mechanical Engineering, Hubei University of Technology, Wuhan 430074, China \\ 3 Department of Mechanical Engineering, Shaoxing University, Shaoxing 312000, China \\ * Correspondence: j112009@foxmail.com; Tel.: +86-575-8834-7502
}

Received: 30 August 2017; Accepted: 28 September 2017; Published: 29 September 2017

\begin{abstract}
Process planning is an important function in a manufacturing system; it specifies the manufacturing requirements and details for the shop floor to convert a part from raw material to the finished form. However, considering only economical criterion with technological constraints is not enough in sustainable manufacturing practice; formerly, criteria about low carbon emission awareness have seldom been taken into account in process planning optimization. In this paper, a mathematical model that considers both machining costs reduction as well as carbon emission reduction is established for the process planning problem. However, due to various flexibilities together with complex precedence constraints between operations, the process planning problem is a non-deterministic polynomial-time (NP) hard problem. Aiming at the distinctive feature of the multi-objectives process planning optimization, we then developed a hybrid non-dominated sorting genetic algorithm (NSGA)-II to tackle this problem. A local search method that considers both the total cost criterion and the carbon emission criterion are introduced into the proposed algorithm to avoid being trapped into local optima. Moreover, the technique for order preference by similarity to an ideal solution (TOPSIS) method is also adopted to determine the best solution from the Pareto front. Experiments have been conducted using Kim's benchmark. Computational results show that process plan schemes with low carbon emission can be captured, and, more importantly, the proposed hybrid NSGA-II algorithm can obtain more promising optimal Pareto front than the plain NSGA-II algorithm. Meanwhile, according to the computational results of Kim's benchmark, we find that both of the total machining cost and carbon emission are roughly proportional to the number of operations, and a process plan with less operation may be more satisfactory. This study will draw references for the further research on green manufacturing in the process planning level.
\end{abstract}

Keywords: process planning; carbon emission; multi-objective optimization; evolutionary algorithm; NSGA-II algorithm

\section{Introduction}

Computer integrated process planning (CAPP) is an important component of a manufacturing system; it bridges the gap between designing and manufacturing. Process planning establishes the technological requirements to convert a part from its raw material to the finished form [1]. CAPP systems optimally determine a feasible process plan for each part such that it can be manufactured economically. Usually, the process planning optimization procedure includes determinations of machines, tools, tool approach directions (TADs), and operation sequence [2]. Nevertheless, the machine, the tool, etc. for an operation is not unique in general; moreover, a given set of operations may have 
different feasible permutations. These flexibilities in both manufacturing resources and operation sequencing increase the difficulty of the problem.

Since the process planning problem is an NP-hard problem, it is difficult to determine the optimal process plan from all the alternatives in polynomial time. Numerous attempts have been paid in process planning optimization to assist human planners in existing research papers. Evolutional and meta-heuristic based methods have largely promoted the development of CAPP technology. These approaches get more research attentions among existing publications. Existing research on process planning optimization mostly pays close attention to one or more conventional goals. For example, Lian et al. [3] proposed a hybrid genetic simulated annealing algorithm in solving the process planning problem. In the algorithm, the genetic algorithm (GA) is treated as the main framework while SA is used as the local search method and machine cost, tool cost, machine change cost, set-up change cost as well as tool change cost were considered in a weighted sum. Later, they investigated the optimization of process planning with various flexibilities, and a novel imperialist competitive algorithm (ICA) is employed to determine promising solutions [4]. Shin et al. also presented a multi-objective symbiotic evolutionary algorithm (MOSEA) for the process planning problem with various flexibilities based on network graphs [5]. Recently, Liu et al. mapped the process planning problem to a weighted graph and they further converted it to a constraint-based traveling salesman problem; the ant colony optimization (ACO) algorithm was adopted to cut down the total cost for machining process [2]. Based on the AND/OR graph, Lv and Qiao proposed new heuristic method, cross entropy (CE) approach, to facilitate the process planning optimization [1]. In addition, process planning in products disassembly has also been considered. Other typical research regarding process planning can be found in [6,7].

Although it seems that the research of process planning is quite fruitful with some significant improvements achieved according to the aforementioned papers, existing work regarding process planning suffers from a limitation: environmental friendliness has never been a major concern in the process planning optimization. Usually, only the economical criteria, such as total machining cost minimization, cannot satisfy the requirement of environment protection nowadays because carbon emission reduction appears especially urgent from a global perspective $[8,9]$. Since greenhouse gases, e.g., carbon dioxide $\left(\mathrm{CO}_{2}\right)$, will be released into the atmosphere when coal-fired power is generated, the consumption of coal-fired electricity by manufacturing sectors will thus indirectly contribute carbon emissions. As pointed in [10], carbon emission can lead to global warming, and manufacturing processes have become a major source of global warming among the various stages of product lifecycle. In many countries, critical environmental regulations enforced enterprises to take carbon emission reduction as a mandatory action. It is undeniable that process planning relates closely with actual manufacturing activities [11]; it comes to our mind that the process planning optimization will play an important role in reducing carbon emission if such criteria are employed in the process planning optimization.

In recent years, researchers have been showing strong interests on carbon emission reduction or energy saving in manufacturing activities and corresponding research is conducted [12-19]. For example, aiming at reducing energy cost to achieve carbon efficiency, Ding et al. developed effective scheduling strategies in a permutation flow shop (PFS) [19]; they suggested a multi-objective $\mathrm{NEH}$ algorithm (MONEH) and a modified multi-objective iterated greedy (MMOIG) algorithm to achieve carbon emission reduction as well as makespan minimization. Hassine et al. [20] adopted a particle swarm optimization (PSO) algorithm for sustainable manufacturing optimization, and a turning procedure case is provided as an example. Since cutting parameters affects machining efficiency and carbon emission, Liu et al. [21] recently proposed a NSGA-II based optimization method to optimize carbon emission cost as well as cutting efficiency; their experimental results reveal that the cutting speed plays a more important role in carbon emission than the feed rate. Recently, Wang et al. [15] developed an estimation of the distribution algorithm with a new decoding method for energy saving in a flexible flow shop environment by optimizing the spindle speed and scheduling scheme 
simultaneously. Meanwhile, Yin et al. [14] tackled a multi-objective single machine scheduling problem that considers total earliness/tardiness minimization as well as energy consumption reduction by their local multi-objective evolutionary algorithm (LMOEA). Nevertheless, these publications consider carbon emission reduction or energy saving by paying attentions to either shop floor scheduling activities or the equipment(component) level instead of the process planning level. We think that carbon emission awareness can also be considered in the process planning stage. Research papers that focus on low carbon emission in process planning are rather limited. Shin et al. [22] developed a green productivity (GP)-based process planning framework where derivations of process parameters for improving GP are allowed; however, carbon emission reduction was not involved in their work. An innovative honey-bee mating and annealing optimization approach is suggested by Li et al. [23] for the integrated process planning and scheduling problem with minimizations of energy consumption and two scheduling criteria; again, carbon emission was not included in their work. Yin et al. [24] gives a novel research on carbon emission reduction in process planning; they developed a methodology to obtain a comparatively 'green' and economical process plan from a micro-process planning perspective. Micro-process planning requires detail procedures and parameters of the implementation of each operation. A representative research that considers carbon emission reduction in process planning is provided by Yi et al. [18] in 2015; corresponding mathematical model considering carbon emission and total processing time is proposed. In their research, the machining procedure of a part is divided into multiple process stages, and, more importantly, the precedence relationship between features depends on the part to be processed; there is no explicit precedence constraints in the proposed model, instead, precedence constraints between features are realized using "rationality constraints", such as "planes prior to its associated features" rule. Different with their research, process plans of a part in this paper is presented by a network graph, where the part is divided into several operations with predefined flexible precedence relationships and each operation has its alternative machining resources. Obviously, network graph representation for flexible process planning is a more general form of other representations. Also, due to the representation of flexible process plans in Yi et al.'s work [18], the other imperfection of their work is that their NSGA-II algorithm can perform process planning optimization for only one part at a time. For the proposed hybrid NSGA-II algorithm in this paper, because of the systematicness of network graph based representations, process planning optimization can be realized for a set of parts simultaneously.

This paper gives a novel mathematical model that considers both total machining coats and carbon emission. The proposed model is developed based on the network graph representation because this representation method can clearly express the flexibilities in process planning and therefore it can bring benefits to mathematical modeling as well as the encoding scheme in applying meta-heuristics especially when there are a set of parts to be processed. After that, a meta-heuristic algorithm, e.g., a hybrid NSGA-II algorithm, is developed to tackle the process planning problem for both carbon emission and machining cost reduction in a Pareto manner. The superiority of the proposed hybrid NSGA-II algorithm over the plain NSGA-II algorithm for process planning is validated through computational experiments using Kim's benchmark. Computational results confirm the effectiveness and efficiency of the proposed algorithm: more promising Pareto fronts have been observed. Compared with existing research papers, the contributions of this paper, summarized as follows, are twofold:

- Due to the swelling stress on the environment protection, in this research carbon emission reduction is integrated into flexible process planning using network graph representations, and mathematical model with explicit operation precedence constraints are introduced into the proposed model. To the best of our knowledge, this is the first research that models the network graph based process planning mathematically.

- Different from other research papers that consider only plain meta-heuristics, a local search method is developed to strengthen the search ability of the algorithm after a study on the characteristics of the process planning problem with low carbon emission concern. The developed problem-specific local search method can force the Pareto front to move to a better direction in the 
solution space. Besides, the TOPSIS method is also adopted to determined the most promising process plan.

The paper therefore proceeds as follows. In the next section, a mathematical model of bi-objective process planning optimization will be put forward. In Section 3, we introduce the hybrid NSGA-II algorithm for the network graph based process planning problem. Experiments with discussions will be reported in Section 4. Section 5 gives the conclusion, limitation and future research directions to finalize this paper.

\section{Mathematical Modeling}

To present a complete set of feasible and alternative manufacturing resources and precedence relationships between operations for each part, jobs to be processed in the process planning problem are represented by a network graph as illustrated in Figure $1 b[5,25,26]$. This network graph represents the manufacturing procedure of a screw given in Figure 1a. Clearly, there are a total of seven features to finish this part. Due to technical constraints, e.g., primary surfaces prior to secondary surfaces and rough machining operations prior to finish machining operations, the example part can be finished with six operations with a certain precedence relationships between operations [26]. One operation is responsible for one or two feature(s); corresponding features are given near the operation in Figure $1 \mathrm{~b}$. In a network graph, both the starting node and the ending node are dummy nodes; they indicate the start and the completion of the manufacturing process of a job, respectively. Dummy nodes have no alternative machines, tools, etc. Other nodes are operation nodes which are marked with operation IDs and OR nodes (marked with 'OR's). An operation node contains the alternative machines that can process the operation, processing times required for the operation and alternative tools. Sometimes, alternative TADs will also be included. For operation 7 in Figure $1 b$, it can be processed by machine 1 or 2 with corresponding processing time 10 or 8 ; meanwhile, it has two candidate tools (2 and 8 ) to complete the processing. The precedence constraints between operations are reflected by arrows: an arrow coming from node A to node B implies that operation B is forced to follow operation A directly or indirectly. An OR node, labeled with "OR", may appear at the bifurcation of two link paths. An OR link path begins at an OR node and ends at it merges with the other OR link path. Operation nodes in exactly one OR link path are needed to be visited. For instance, there is an OR node in Figure $1 \mathrm{~b}$ and this means either operations 1,3 or operations 2,4 should be selected to complete the manufacturing. Further, if a bifurcation is not marked with an OR node, operations belonging to both OR link paths should be visited.

The complexity involved in process planning stems from three kinds of flexibilities: operation flexibility (OF), sequencing flexibility (SF) and processing flexibility (PF). OF refers to determining a specific machine and a tool for an operation. PF refers to the alternative operations in OR link paths to complete the same manufacturing feature. With SF, various feasible operation permutations, which satisfies precedence constraints, are allowed. Obviously, such flexibilities make the decision procedure much more complex in process planning.

In this section, a mathematical model is established for the process planning problem to reduce machining costs as well as carbon emission. In our previous study [27], the process planning problem and the job shop scheduling problem are considered simultaneously, and, more importantly, feasible operation permutations in process planning are achieved by putting process planning into the context of job shop scheduling with the help of established pre-ordered sets and back-ordered sets. Different from our previous study, however, process planning does not cling to the job shop scheduling any more in this case; a position based modeling strategy is thus adopted in the proposed model. Some notations are first described as follows. 


\section{b)}

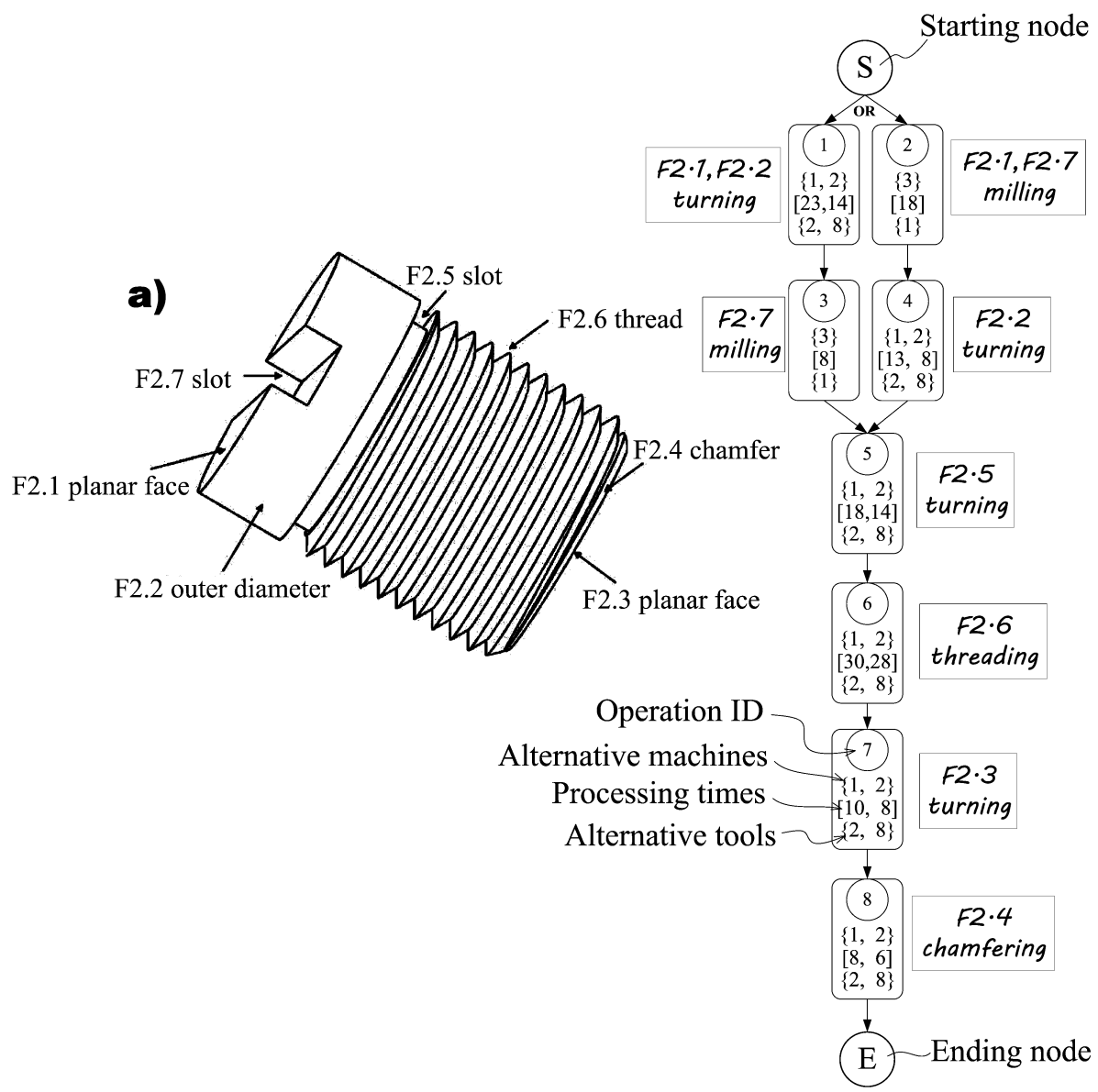

Figure 1. Network graph representations: (a) an example part; and (b) a network graph of the example part in process planning.

\section{Subscripts and Notations}

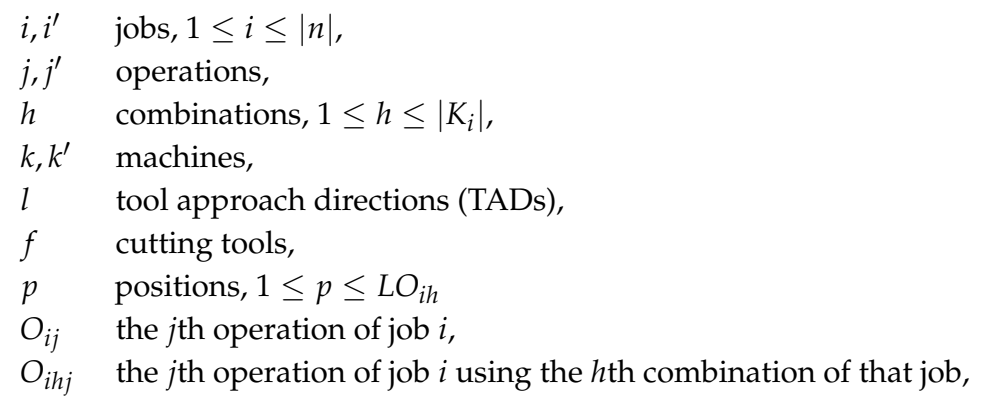

\section{Sets and Parameters}

$K_{i} \quad$ the set of combinations of job $i$,

$n$ the set of jobs,

$M_{i j} \quad$ the set of available machines for $O_{i j}$,

$R_{i h} \quad$ the set that contains the operations belonging to the $h$ th combination of job $i$,

$T_{i j} \quad$ the set of available cutting tools of operation $O_{i j}$,

$T A D_{i j}$ the set of TADs of operation $O_{i j}$,

$L O_{i h} \quad$ the number of operations of of the $h$ th combination of job $i$, e.g., $L O_{i h}=\left|R_{i h}\right|$,

$P W_{k} \quad$ the power of machine $k$,

$t_{i j k} \quad$ the processing time of operation $O_{i j}$ on machine $k$, 


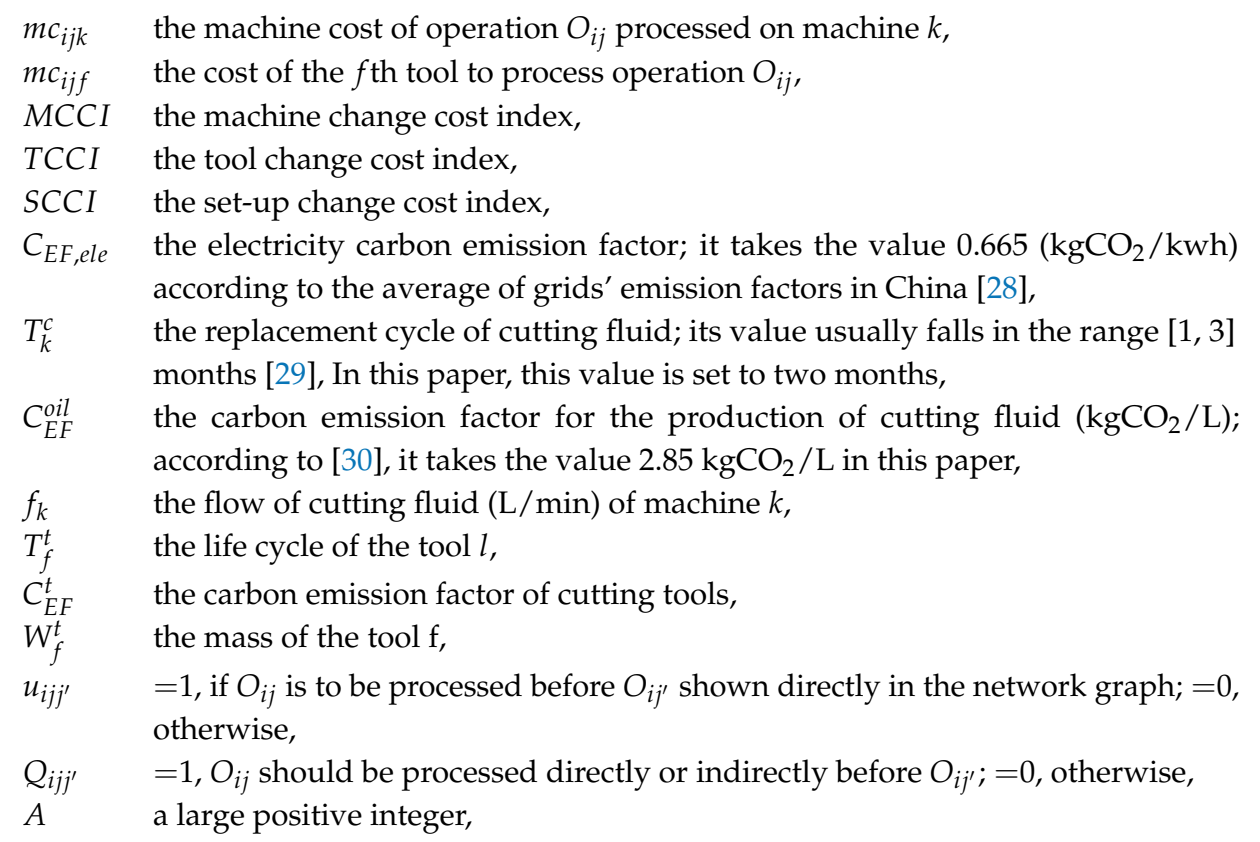

\section{Variables}

$Y_{i h}=1$, if the $h$ th combination of job $i$ is selected; $=0$, otherwise,

$X_{i h j k}=1$, if the operation $O_{i h j}$ is processed on machine $k$; $=0$, otherwise,

$x_{i h j p}=1$, if the operation $O_{i h j}$ is processed on the $p$ th position; $=0$, otherwise,

$Z_{\text {ihjf }}=1$, if the operation $O_{i h j}$ is processed by tool $f ;=0$, otherwise,

$V_{i h j l}=1$, if the $l$ th TAD is applied in processing the operation $O_{i h j} ;=0$, otherwise,

$S_{i j j^{\prime}}=1$, if operation $O_{i j}$ is processed directly or indirectly before $O_{i j^{\prime}} ;=0$, otherwise,

\section{Objectives}

The first objective of this research is to minimize the machining costs. Usually, machining costs consist of machine cost (MC), tool cost (TC), machine change cost (MCC), tool change cost (TCC) and set-up cost (SC) $[4,31]$. The first objective can thus be regarded as the total weighted cost (TWC) by summing up the weighted costs mentioned above; and this objective can be expressed as follows:

$$
T W C=w_{1} M C+w_{2} T C+w_{3} M C C+w_{4} T C C+w_{5} S C
$$

These weights, e.g., $w_{1} \sim w_{5}$, are set to 1 in our research for convenience, and this means that all kinds of costs are treated equally important. Five kinds of costs in Equation (1) can be calculated using Equations (2)-(6) as follows:

$$
\begin{aligned}
M C & =\sum_{i \in n} \sum_{h \in K_{i}} \sum_{j \in R_{i h}} \sum_{k \in M_{i j}} m c_{i j k} X_{i h j k} \\
T C & =\sum_{i \in n} \sum_{h \in K_{i}} \sum_{j \in R_{i h}} \sum_{f \in T_{i j}} m c_{i j f} Z_{i h j f}
\end{aligned}
$$

If two adjacent operations of the same job are to be processed with different machines, tools and TADs, machine change cost, tool change cost and set-up cost will be taken into account. Machine change cost, triggered when two adjacent operations are processed by different machines, is defined in Equation (4). It is clear that if operation $O_{i h j}$ is processed by machine $k$ in the $p$ th position, the term $X_{i h j k}+x_{i h j p}$ takes the value 2; more precisely, the term $\left\lfloor\left(X_{i h j k}+x_{i h j p}+X_{i h j^{\prime} k}+x_{i h j^{\prime}, p+1}\right) / 4\right\rfloor$ in Equation (4) takes the value 1 if two adjacent operations, $O_{i h j}$ and $O_{i h j^{\prime}}$ in this case, are processed by the same machine (machine $k$ ). 


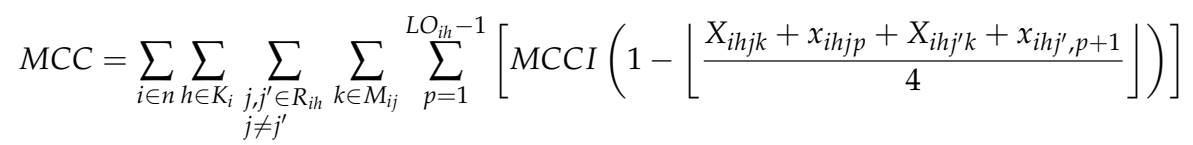

Whenever two consecutive operations are not performed on the same machine or using the same tool, a tool change happens; corresponding costs are given in Equation (5). Similarly, the term $\left(X_{i h j k}+x_{i h j p}+Z_{i h j f}+X_{i h j^{\prime} k}+x_{i h j^{\prime}, p+1}+Z_{i h j^{\prime} f}\right)$ takes the value 6 if two adjacent operations of the same job are processed on the same machine with the same tool.

$$
\text { TCC }=\sum_{i \in n} \sum_{h \in K_{i}} \sum_{\substack{j, j^{\prime} \in R_{i h} \\ j \neq j^{\prime}}} \sum_{k \in M_{i j}} \sum_{p=1}^{L O_{i h}-1} \sum_{f \in T_{i j}}\left[\operatorname{TCCI}\left(1-\left\lfloor\frac{X_{i h j k}+x_{i h j p}+Z_{i h j f}+X_{i h j^{\prime} k}+x_{i h j^{\prime}, p+1}+Z_{i h j^{\prime} f}}{6}\right\rfloor\right)\right]
$$

Finally, a set-up change happens if two adjacent operations are not processed on the same machine with the same TAD. With the set-up change cost index, , set-up change cost can be calculated using Equation (6).

$$
S C C=\sum_{i \in n} \sum_{h \in K_{i}} \sum_{\substack{j, j^{\prime} \in R_{i h} \\ j \neq j^{\prime}}} \sum_{k \in M_{i j}} \sum_{p=1}^{L O_{i h}-1} \sum_{l \in T A D_{i j}}\left[S C C I\left(1-\left\lfloor\frac{X_{i h j k}+x_{i h j p}+V_{i h j l}+X_{i h j^{\prime} k}+x_{i h j^{\prime}, p+1}+V_{i h j^{\prime} l}}{6}\right\rfloor\right)\right]
$$

The second objective lays stress on environment protection by minimizing carbon emissions. A manufacturing system often involves more than one machines, and machine tools are the particular source of carbon emissions since the amount of electricity that powers the machines is generated through power plants. Relative research $[28,30]$ has already shown that carbon emission is induced by a wide range of activities. To reflect the nature of carbon emission in machining process, quantitative measures of carbon emissions are considered in the second objective.

As shown in Equation (7), the total quantized carbon emissions $\left(C_{E i}, \mathrm{kgCO}_{2}\right)$ consist of three parts: the carbon emission caused by the electricity generation for machining process $\left(C_{E i}^{e}, \mathrm{kgCO}_{2}\right)$, the carbon emission caused by the production of cutting fluid $\left(C_{E i}^{c}, \mathrm{kgCO}_{2}\right)$, and the carbon emission caused by the production of tools $\left(\mathrm{C}_{E i}^{t}, \mathrm{kgCO}_{2}\right)$ :

$$
C_{E i}=C_{E i}^{e}+C_{E i}^{c}+C_{E i}^{t}
$$

In fact, the carbon emission caused by the electricity generation for machining process $\left(C_{E i}^{e}\right)$ consists two parts: the carbon emission caused by the energy consumption in cutting process $\left(E_{C i}^{c u t}\right)$ and the one caused by the auxiliary energy consumption $\left(E_{C i}^{a u}\right)$. Since the auxiliary energy consumption takes only a small proportion of the whole energy consumption, in this research, only the carbon emission caused by the energy consumption in cutting process is considered. Thus, the carbon emissions result from the electricity consumption for machining process can be calculated by the following form:

$$
C_{E i}^{e}=C_{E F, e l e} E_{C i}^{c u t}=C_{E F, e l e} \sum_{i \in n} \sum_{h \in K_{i}} \sum_{j \in R_{i h}} \sum_{k \in M_{i j}} X_{i h j k} t_{i j k} P W_{k}
$$

For the carbon emission caused by the production of cutting fluid, $C_{E i}^{c}$, it can be calculated as follows:

$$
C_{E i}^{c}=\sum_{i \in n} \sum_{h \in K_{i}} \sum_{j \in R_{i h}} \sum_{k \in M_{i j}} X_{i h j k} \frac{t_{i j k}}{T_{k}^{c}} C_{E F}^{o i l}\left(0.5 \% f_{k} t_{i j k}\right)
$$

Since the water based cutting fluid is a mixture of water and mineral oil, and the volume ratio of mineral oil is about 5\%, the volume of cutting fluid as shown in Equation (9) can thus be calculated as $0.5 \% f_{k} t_{i j k}$. As a tractable manner, we assume that the flow of cutting fluid, $f_{k}$, is proportionate to the power of the machine tool and it varies in the interval [5 L/min, $20 \mathrm{~L} / \mathrm{min}]$. 
The last term in Equation (7) corresponds to the carbon emission caused by the production of tools. This can be quantized using Equation (10).

$$
C_{E i}^{t}=\sum_{i \in n} \sum_{h \in K_{i}} \sum_{j \in R_{i h}} \sum_{f \in T_{i j}} Z_{i h j f} \frac{t_{i j k}}{T_{f}^{t}} C_{E F}^{t} W_{f}^{t}
$$

Usually, the value of the life cycle of tool $f$, $T_{f}^{t}$, falls within the interval [60 $\mathrm{min}, 240 \mathrm{~min}$ ]; in this paper, it takes the value $150 \mathrm{~min}$. The carbon emission factor of cutting tools, $C_{E F}^{t}$, takes the value $104.6 \mathrm{kgCO}_{2} / \mathrm{kg}$. Finally, according to [28,32], the average mass of a tool cutter is $9.5 \mathrm{~g}$.

\section{Constraints}

It is rather tedious and error-prone to identify all the possible operation permutations from a network graph. It can be observed from a network graph that combinations, which contain indispensable operations for finishing the job, can be identified by judging the operations in corresponding OR link paths in a network graph. Thus, a feasible operation permutation can be obtained by properly permuting the operations in a combination. As illustrated in Figure $2 a-c$, this job has two operation combinations, as shown in Figure $2 b, c$, by judging two OR link paths. One and only one of the two combination should be selected to complete the whole manufacturing procedure. In the worst case, the maximum possible number of combinations is $2^{N}$ ( $N$ is the number of OR nodes in a network graph); nevertheless, since the number of OR nodes is rather limited and one OR node is often included in the other one, there are few combinations in a network graph. For instance, $(1 \rightarrow 7 \rightarrow 8 \rightarrow 2 \rightarrow 4 \rightarrow 5 \rightarrow 3 \rightarrow$ $9 \rightarrow 10 \rightarrow 6 \rightarrow 11 \rightarrow 14)$ and $(7 \rightarrow 1 \rightarrow 2 \rightarrow 4 \rightarrow 8 \rightarrow 5 \rightarrow 12 \rightarrow 3 \rightarrow 13 \rightarrow 14 \rightarrow 6)$ are two possible resultant process plans (operation permutations) dominated by the combinations in Figure $2 b, c$ respectively. The basic idea in modeling the process planning problem is to determine correct precedence relationships for the operations in a combination with corresponding constraints as well as objectives.

The first constraint set specifies that exactly one combination should be selected for each job.

$$
\sum_{h \in K_{i}} Y_{i h}=1, \forall i \in n
$$

Further, the operations, which belong to the selected combination, should be assigned to exactly one available machine; otherwise, the operations in an unselected combination will not be assigned to a machine.

$$
\sum_{k \in M_{i j}} X_{i h j k}=Y_{i h}, \forall i \in n, \forall h \in K_{i}, \forall j \in R_{i h}
$$

Any operations in a selected combination can only be assigned to a position. For instance, operation 9 in Figure $2 \mathrm{~d}$ has been assigned to the fifth position and it cannot be assigned to any other positions. This can be ensured by introducing a constraint set as follows:

$$
\sum_{p=1}^{L O_{i h}} x_{i h j p}=Y_{i h}, \forall i \in n, \forall h \in K_{i}, \forall j \in R_{i h}
$$

Similarly, a given position can accommodate one and only one operation as shown in constraint set Equation (14):

$$
\sum_{j \in R_{i h}} x_{i h j p}=Y_{i h}, \forall i \in n, \forall h \in K_{i}, \forall p \in\left[1, L O_{i h}\right]
$$

The following constraint sets relate to the precedence relationships between operations to obtain a proper operation permutation. Based on the parameters, $u_{i j j^{\prime}}$, the precedence relationship between any two operations can be established using Algorithm 1 discussed in our previous study [27]. 


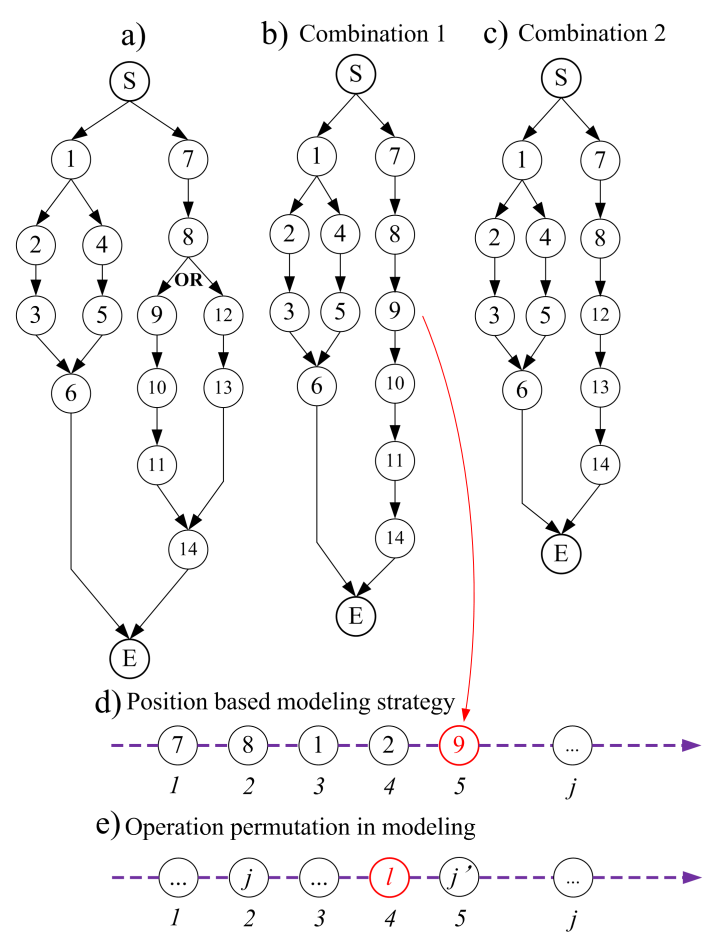

Figure 2. Operation combinations with modeling strategy: (a) an example network graph for process planning; (b,c) two combinations; and (d,e) modeling strategies.

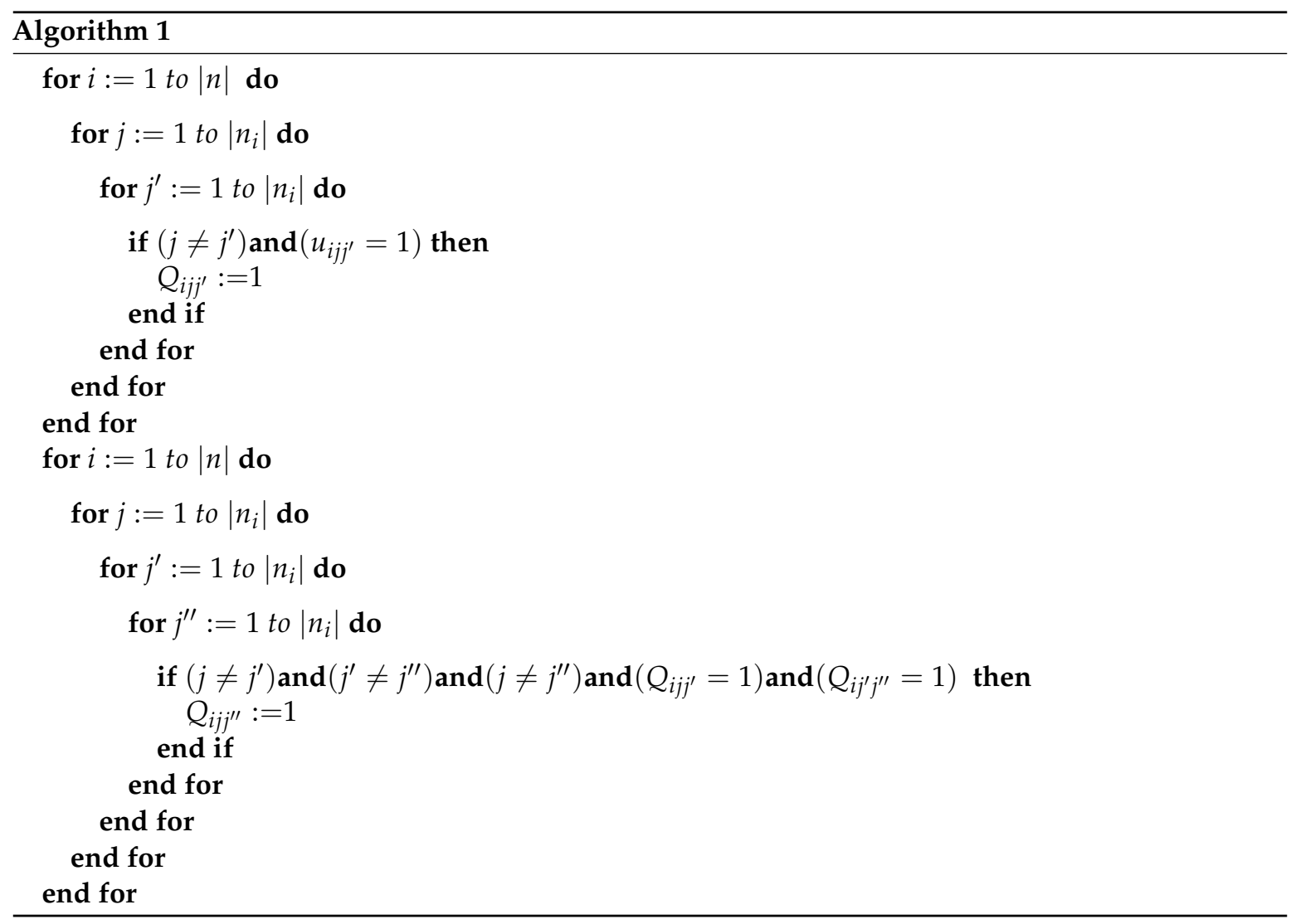

Obviously, the parameter $Q_{i j j^{\prime}}$ equals 1 if operation $O_{i j^{\prime}}$ follows $O_{i j}$ directly or indirectly. If $Q_{i j j^{\prime}}+Q_{i j^{\prime} j}=0$, there is no restriction on the precedence relationship of the two operations. 
According to Figure 2e, the decision variable $x_{i h j p}$ takes the value 1 before the variable $x_{i h j^{\prime}}$, if $Q_{i j j^{\prime}}=1$ and the $h$ th combination is selected. Thus, the following constraint set is introduced.

$$
\sum_{p=1}^{l} x_{i h j p} \geq \sum_{p=1}^{l} x_{i h j^{\prime} p}-A\left(1-Q_{i j j^{\prime}}\right)-A\left(1-Y_{i h}\right), \forall i \in n, \forall h \in K_{i}, \forall j, j^{\prime} \in R_{i h}, j \neq j^{\prime}, \forall l \in\left[1, L O_{i h}\right]
$$

Besides, operations that have no precedence relationships with other operations exist; for instance, either one of operations 2 and 4 in Figure 2a can be processed before the other one. Constraint set Equation (15) is powerless to restrict the precedence relationship between such operations, and a set of $0-1$ variables $S_{i j j^{\prime}}$ is introduced to determine a proper precedence relationship between two such operations. Constraint sets Equations (16) and (17) are equal to $S_{i j j^{\prime}}+S_{i j^{\prime} j}=1$ provided that $Q_{i j j^{\prime}}+Q_{i j^{\prime} j}=0$.

$$
\begin{aligned}
& S_{i j j^{\prime}}+S_{i j^{\prime} j} \geq 1-A\left(Q_{i j j^{\prime}}+Q_{i j^{\prime} j}\right), \quad \forall i \in n, \forall j, j^{\prime}, j \neq j^{\prime} \\
& S_{i j j^{\prime}}+S_{i j^{\prime} j} \leq 1+A\left(Q_{i j j^{\prime}}+Q_{i j^{\prime} j}\right), \quad \forall i \in n, \forall j, j^{\prime}, j \neq j^{\prime}
\end{aligned}
$$

After this, precedence constraints similar with constraint set Equation (15) can be established to facilitate a feasible operation permutation:

$$
\begin{aligned}
& \sum_{p=1}^{l} x_{i h j p} \geq \sum_{p=1}^{l} x_{i h j^{\prime} p}-A\left(1-S_{i j j^{\prime}}\right)-A\left(1-Y_{i h}\right), \quad \forall i \in n, \forall h \in K_{i}, \forall j, j^{\prime} \in R_{i h}, j \neq j^{\prime}, \forall l \in\left[1, L O_{i h}\right] \\
& \sum_{p=1}^{l} x_{i h j^{\prime} p} \geq \sum_{p=1}^{l} x_{i h j p}-A\left(1-S_{i j^{\prime} j}\right)-A\left(1-Y_{i h}\right), \quad \forall i \in n, \forall h \in K_{i}, \forall j, j^{\prime} \in R_{i h}, j \neq j^{\prime}, \forall l \in\left[1, L O_{i h}\right]
\end{aligned}
$$

The subscript of jobs, $i$, can be omitted provided that there is only one job in the problem. Due to the complexity of the proposed model, the hybrid NSGA-II algorithm is developed to address this problem.

\section{The Hybrid NSGA-II Algorithm}

\subsection{Encoding Scheme}

The network graph-specific coding scheme is designed as illustrated in Figure 3. An individual contains process plans of all the jobs. For each job, a feasible permutation of operations together with corresponding machines is presented; the last position of the process plan is the combination ID that indicates which combination is selected. For example, the first job(Job 1) in Figure 3 corresponds to the job in Figure 2a with a feasible operation permutation $(7 \rightarrow 8 \rightarrow 1 \rightarrow 12 \rightarrow 2 \rightarrow 4 \rightarrow 5 \rightarrow 13 \rightarrow 3 \rightarrow$ $14 \rightarrow 6$ ), and these operations belong to the second combination. Unfortunately, alternative tools and TADs are not given in Kim's benchmark; thus, such information is not reflected in the encoding scheme in Figure 3. However, this encoding scheme does not hinder the application of the proposed hybrid NSGA-II algorithm: alternative tools and TADs can also be added to each operation in an individual. Initially, operation permutation of each job is randomly generated and the binary tree method [33] is applied to obtain a feasible operation permutation for each job.

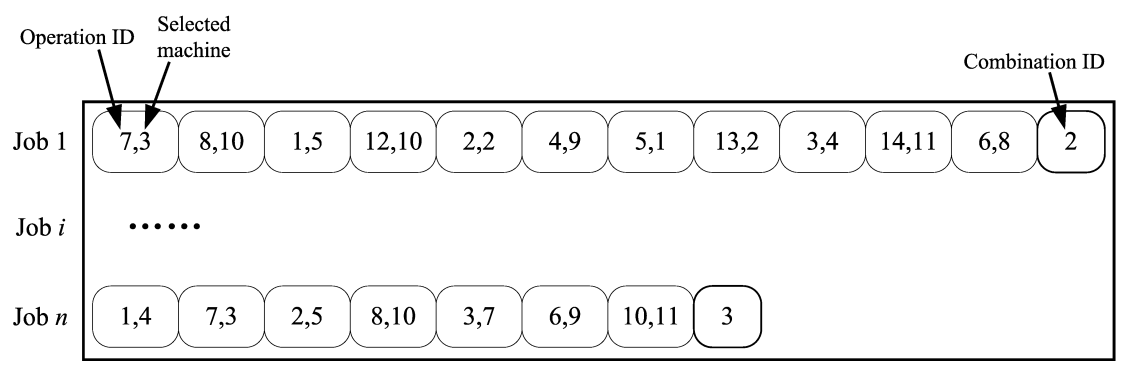

Figure 3. The structure of an individual. 


\subsection{Genetic Operators}

In the crossover operator, several jobs are first determined randomly and corresponding operation permutations together with selected machines and combination IDs of the selected jobs are exchanged with the ones in the other selected individual. After that, the second crossover operator can be applied to take advantage of sequencing flexibility (SF): if two operation permutations of the same job in two individuals have the same combination ID (but not necessarily with the same operation permutation), the single point crossover procedure [34] is performed. This single point crossover procedure ensures the feasibility of operation permutations. For the mutation procedure, it is realized by changing a machine for an randomly selected operation.

\subsection{The Local Search Method}

The proposed local search algorithm considers both the two objectives, and it integrates problem specific knowledge into the search process. For each operation in a process plan, alternative machines belonging to the operation are tried; a machine will be selected for the operation when an improvement is observed. If one of the following two cases happens, the new machine for the operation is accepted.

- Both total machining costs and the carbon emission are improved by the local search method

- One criterion is improved and the other one performs not worse than before.

The algorithm stops if there is not improvement observed. Algorithm 2 describes the whole local search procedure.

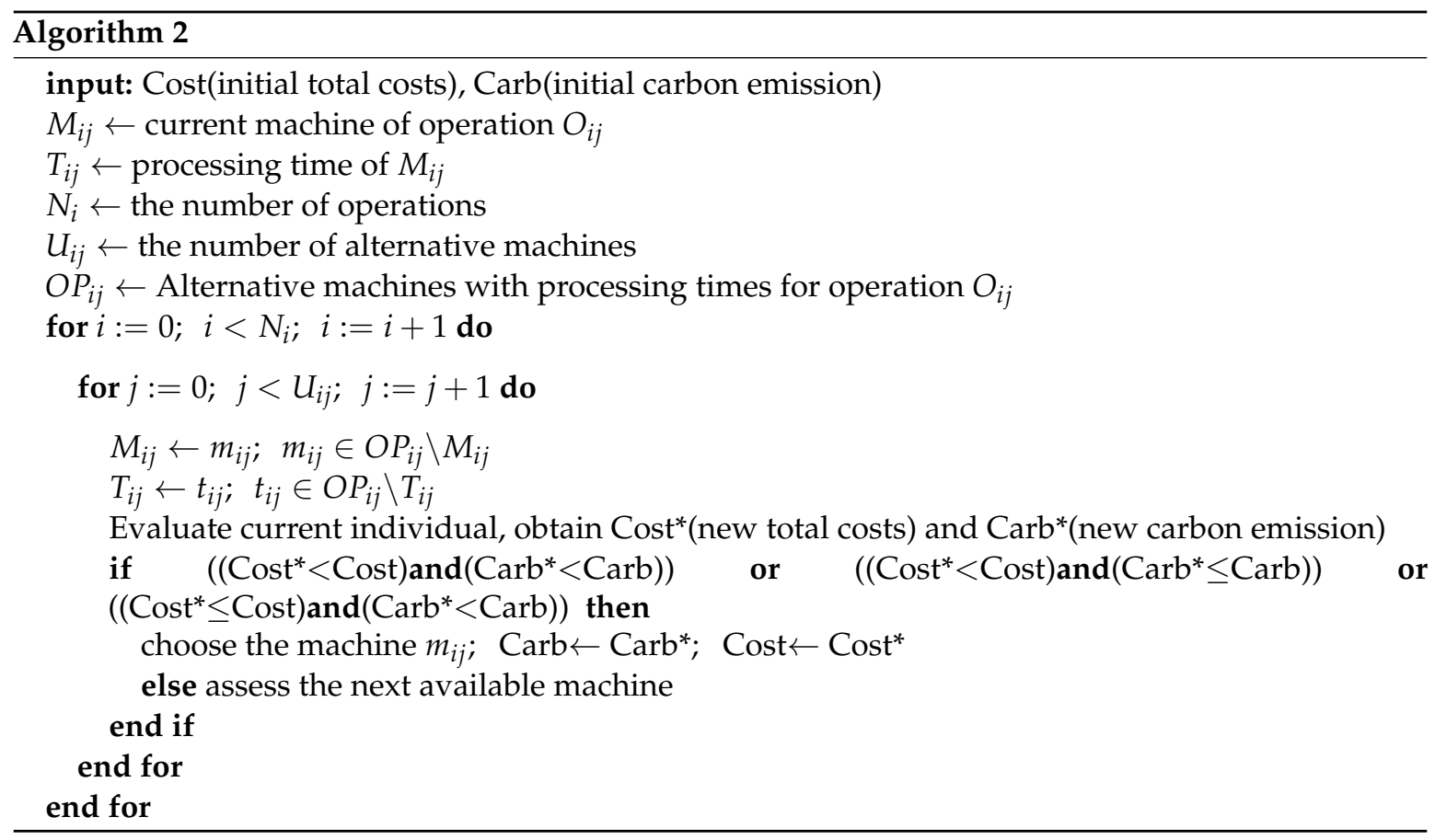

\subsection{The Outline of Hybrid NSGA-II}

Following the method proposed by Deb et al. [35], the algorithm adopts the non-dominated sorting technique to sort all the solutions in $P_{t}$ and $Q_{t}$. This fast sorting method favors both the population diversity as well as the fast convergence ability. After that, the crowding distance technique is applied and a certain number of individuals are selected for the next generation based on rankings and crowding distances. After the last iteration, the TOPSIS method is applied. This decision supporting strategy chooses the solution in the optimal Pareto front with shortest distance from the ideal solution. Since the two criteria are equally important, they are assigned with equal weights $\left(w_{i}=0.5, i=1,2\right)$. Figure 4 gives the workflow of the proposed algorithm. 


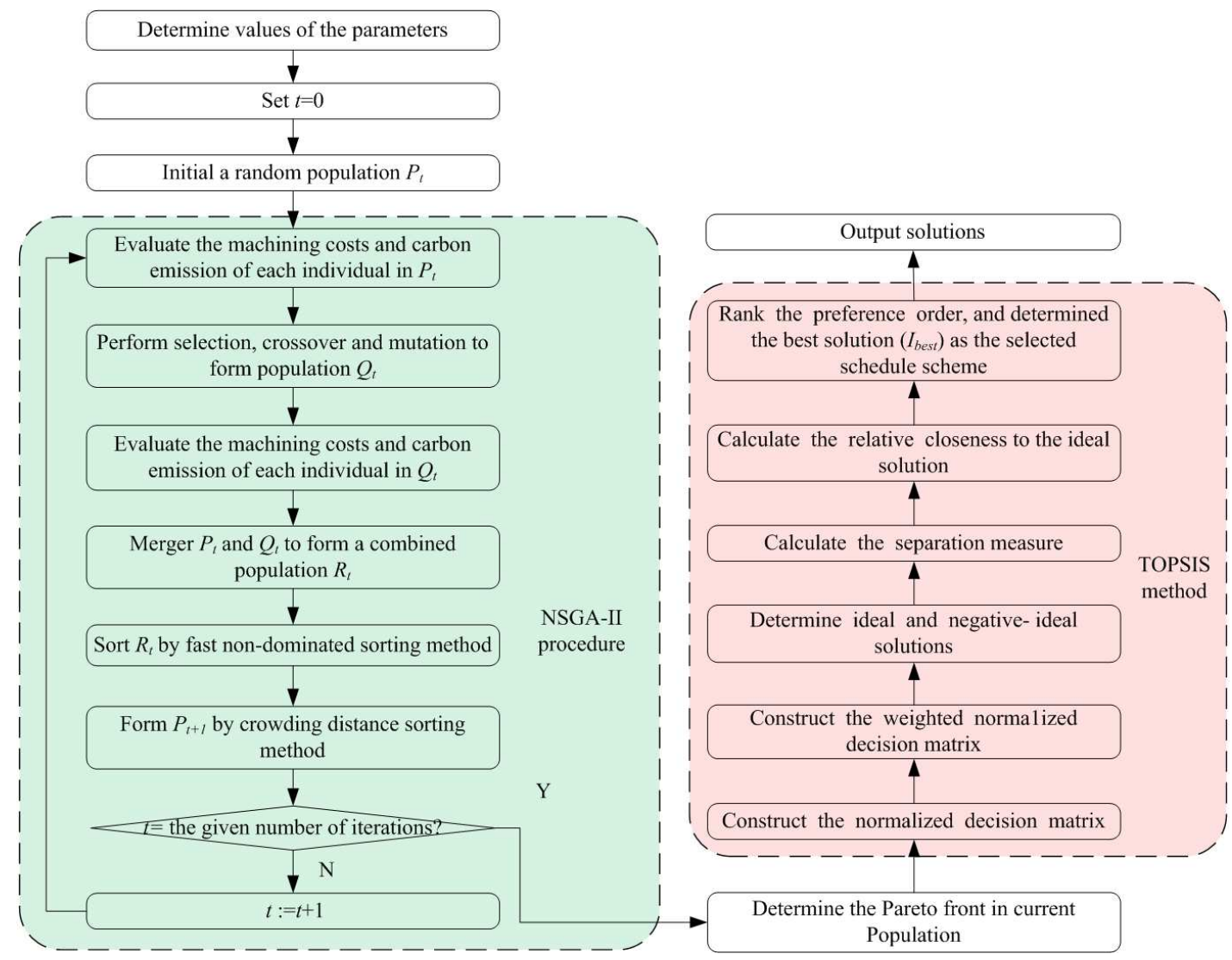

Figure 4. The workflow of hybrid NSGA-II.

\section{Experiments}

To evaluate the performance of the developed algorithm and obtain the process plans with low costs and carbon emission, typical benchmark, Kim's benchmark [25], is adopted. We compare the best Pareto front obtained by the proposed hybrid NSGA-II algorithm and the plain NSGA-II algorithm to demonstrate the advantage of the hybrid NSGA-II algorithm over the plain NSGA-II algorithm. Moreover, the ideal solution is also provided. Both the two algorithms are coded in $\mathrm{C}++$ language and implemented on a PC with an Intel i5 $34703.2 \mathrm{GHz}$ CPU with 4 GB of RAM. In our algorithm, the unit of processing times in Kim's benchmark is minute. Based on the actual situations, powers of 15 machine tools are sampled in the interval [ $3 \mathrm{kw}, 30 \mathrm{kw}]$ and corresponding machine powers are presented in Table 1. Both algorithms are performed with the same parameters; population size and the number of iteration are set to 500 and 400, respectively. In addition, the crossover probability and the mutation probability are set to 0.7 and 0.05 .

Table 1. Machine power used in computation.

\begin{tabular}{cccccc}
\hline Machine ID & Power $(\mathbf{k W})$ & Machine ID & Power $\mathbf{( k W )}$ & Machine ID & Power (kW) \\
\hline 1 & 25 & 6 & 19 & 11 & 7 \\
2 & 12 & 7 & 7 & 12 & 21 \\
3 & 17 & 8 & 5 & 13 & 9 \\
4 & 18 & 9 & 23 & 14 & 13 \\
5 & 12 & 10 & 16 & 15 & 28 \\
\hline
\end{tabular}


The optimal Pareto fronts obtained by hybrid NSGA-II and the plain NSGA-II algorithm are compared. Depending on the problem indexes, the number of jobs in an instance in Kim's benchmark ranges from 6 to 18; hence, the complexity increases as the instance index increases. Pareto fronts taken from Small, medium and large scale instances are compared.

As presented in Figure 5, both the Pareto fronts obtained by the two algorithms are distributed very equally in the solution space; however, Pareto fronts obtained by hybrid NSGA-II are much better than those obtained by plain NSGA-II because non-dominated solutions obtained by hybrid NSGA-II occupy more favorable positions according to Figure 5. The reason behind this lies in that problem specific local search method is developed in hybrid NSGA-II, and high effectiveness is observed in this case. Due to the limitation of the problem scale, the improvement of the hybrid NSGA-II algorithm is somewhat limited in solving the first instance (a small scale instance). Thus, the two Pareto fronts of Instance 1 in Figure 5a get very close. For other cases, especially for large scale instances, the optimization efficiency of the proposed hybrid NSGA-II is quite remarkable. In general, solutions obtained by hybrid NSGA-II are always better than the ones obtained by the plain NSGA-II algorithm. Therefore, this indicates that integrating problem-specific local search methods into a meta-heuristic can obtain more competitive solutions.
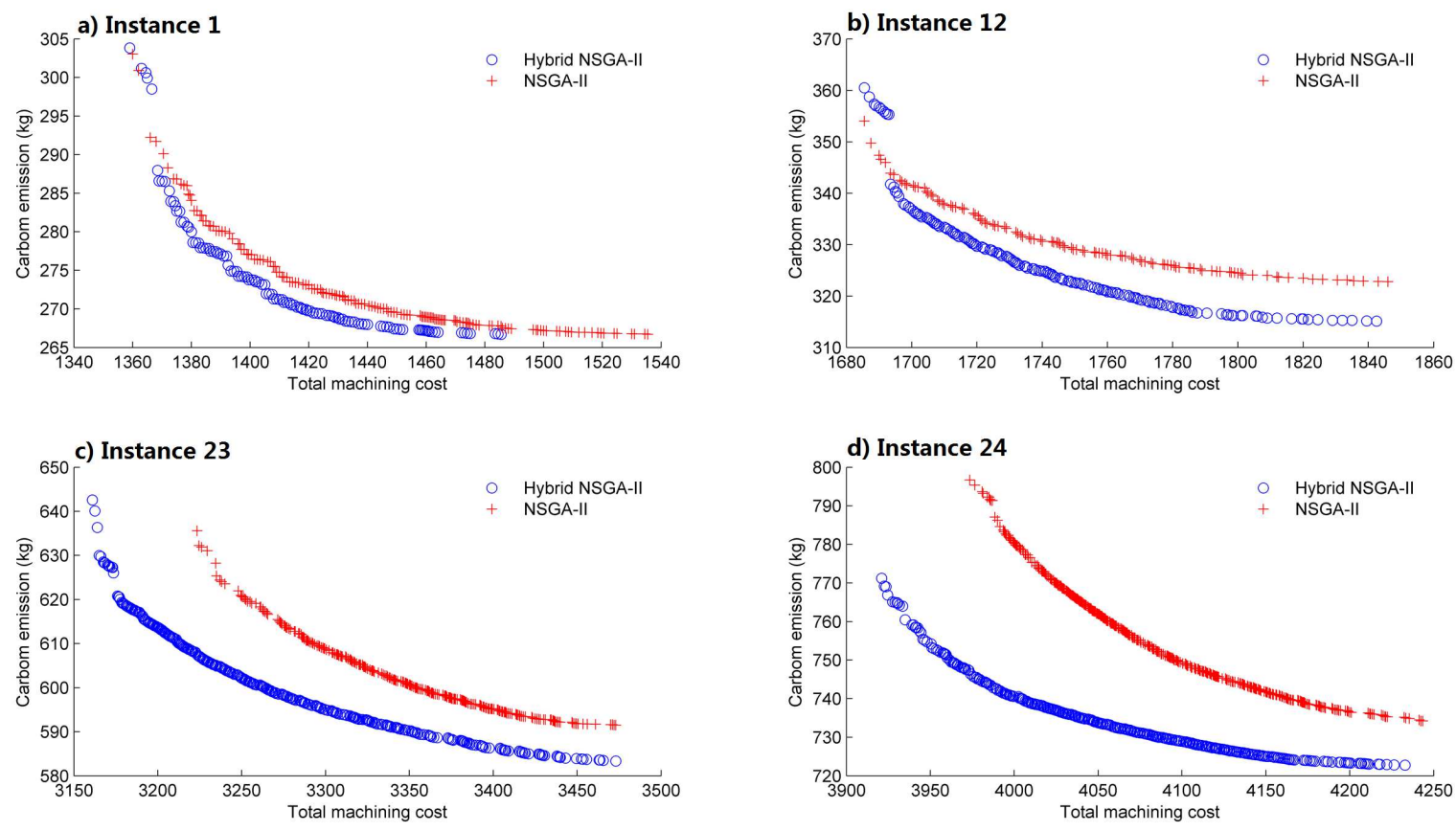

Figure 5. Comparisons of Pareto fronts.

After the last iteration, the ideal solution of each instance is determined by the TOPSIS method. Corresponding ideal solutions are listed in Table 2. In Table 3, the detailed information of the ideal solution of the first instance is presented. It can be seen that the first instance consists of six jobs (jobs 1, 2, 3, 10, 11 and 12) and operation permutations with machine assignments of jobs in this instance certainly meet the requirements and precedence relationships specified in corresponding network graphs of Kim's benchmark. 
Table 2. Ideal solutions of each instance.

\begin{tabular}{ccccc}
\hline Instances & Jobs & Operations * & Total Cost & Carbon Emission $(\mathbf{k g})$ \\
\hline 1 & 6 & 79 & 1408 & 271.313 \\
2 & 6 & 100 & 1257.5 & 232.638 \\
3 & 6 & 121 & 1319 & 224.852 \\
4 & 6 & 95 & 1163 & 201.197 \\
5 & 6 & 96 & 1187.5 & 233.019 \\
6 & 6 & 109 & 1627 & 297.93 \\
7 & 6 & 99 & 1405 & 289.384 \\
8 & 6 & 96 & 1348 & 236.192 \\
9 & 6 & 105 & 1245.5 & 207.114 \\
10 & 9 & 132 & 2073.5 & 392.393 \\
11 & 9 & 168 & 1916 & 339.805 \\
12 & 9 & 146 & 1746.5 & 323.099 \\
13 & 9 & 154 & 2257.5 & 405.518 \\
14 & 9 & 151 & 2107 & 407.426 \\
15 & 9 & 149 & 1885 & 329.587 \\
16 & 12 & 179 & 2653.5 & 506.928 \\
17 & 12 & 221 & 2599.5 & 462.087 \\
18 & 12 & 191 & 2341 & 434.744 \\
19 & 12 & 205 & 2846.5 & 529.171 \\
20 & 12 & 195 & 2749 & 526.667 \\
21 & 12 & 201 & 2606.5 & 440.523 \\
22 & 15 & 256 & 3367 & 609.431 \\
23 & 15 & 256 & 3255.5 & 601.093 \\
24 & 18 & 300 & 3996 & 740.992 \\
\hline$*$
\end{tabular}

* the sum of operations contained in network graphs of jobs in an instance.

Table 3. Operation information of Instance 1.

\begin{tabular}{cl}
\hline Jobs & Operation Information \\
\hline 1 & $(1,14)(2,11)(5,13)(3,15)(4,12)(6,12)(7,11)(8,8)$ \\
2 & $(7,11)(8,10)(1,8)(12,8)(2,8)(3,4)(4,7)(5,7)(13,7)(6,5)(14,10)$ \\
3 & $(1,11)(2,11)(16,10)(17,10)(7,5)(13,14)(8,1)(9,13)(10,13)(11,2)(14,2)(5,2)(18,7)(3,7)(6,7)(4,7)(19,8)(15,8)(12,14)$ \\
10 & $(3,13)(4,13)(5,7)(6,5)(9,2)(1,2)(10,3)(11,14)(2,15)$ \\
11 & $(1,6)(2,8)(6,2)(8,2)(7,14)(3,13)(4,13)(9,13)(5,6)$ \\
12 & $(1,11)(2,8)(3,5)(4,12)(5,15)(13,3)(8,3)(9,6)(16,7)(17,4)(10,4)(11,7)(6,13)(18,13)(12,10)(7,11)$ \\
\hline & The first number in parentheses denotes the operation ID and the second is the machine ID.
\end{tabular}

In general, total costs and carbon emission are not completely independent variables according to Table 2. The least square fitting method is applied to study the relationship between carbon emission and the number of operations (the sum of operations in an instance). Figure 6 gives the resultant fitting curve; with more operations carbon emission becomes more prone to increase. In Figure 6, it can be seen that there is a roughly linear relationship between total carbon emission and the number of operations. According to the gradient of the curve, with one more operation, the carbon emission will increase $2.3 \mathrm{~kg}$ in average.

Similarly, the relationship between total cost and the number of operations is also studied and fitting curve is given in Figure 7. There is still a proportional relationship between total cost and the number of operations. The gradient of the curve in Figure 7 shows that with one more operation, the total cost will increase about 12.66 units. Based on the analysis above, one should choose a process plan with less operations under the same condition to save machining cost and avoid carbon emission. 


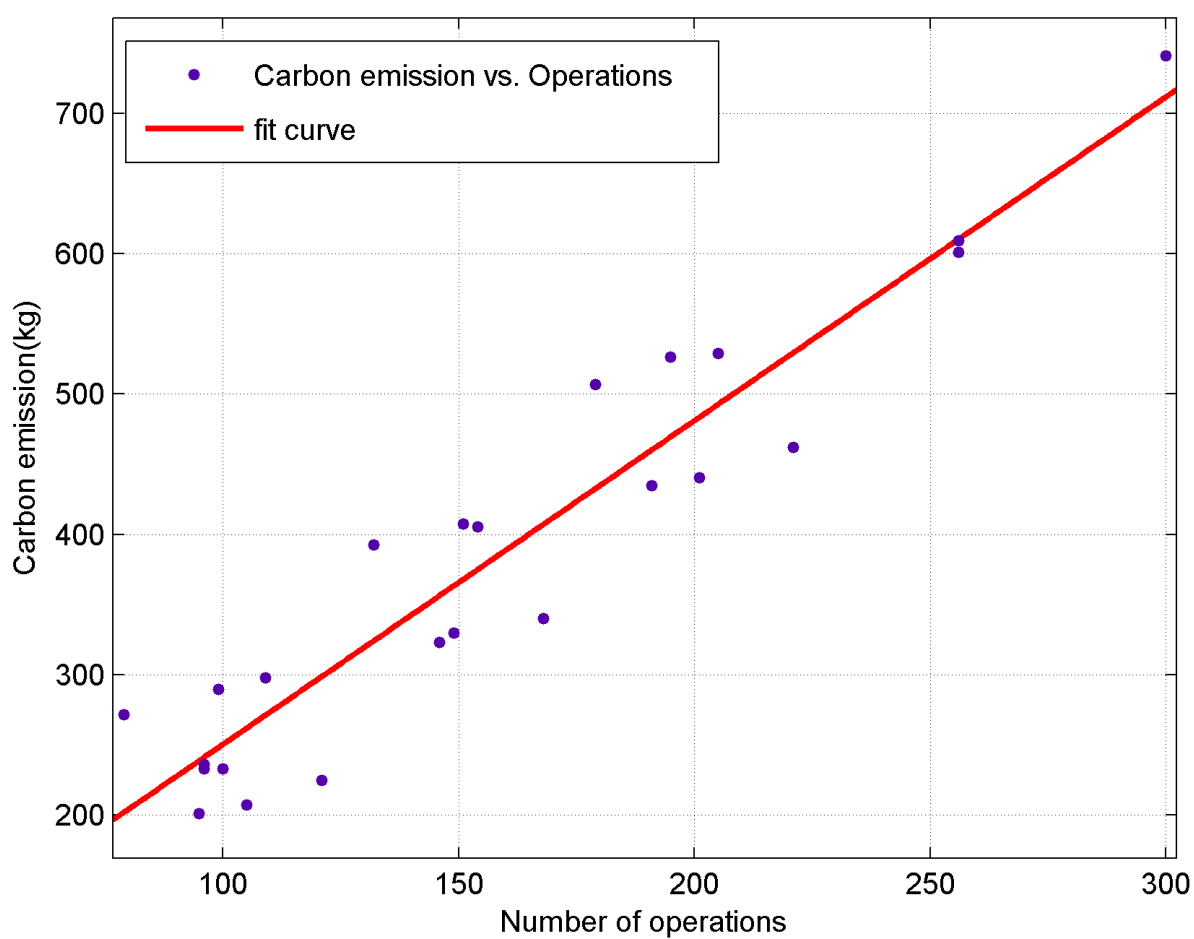

Figure 6. Relationship between carbon emission and the number of operations.

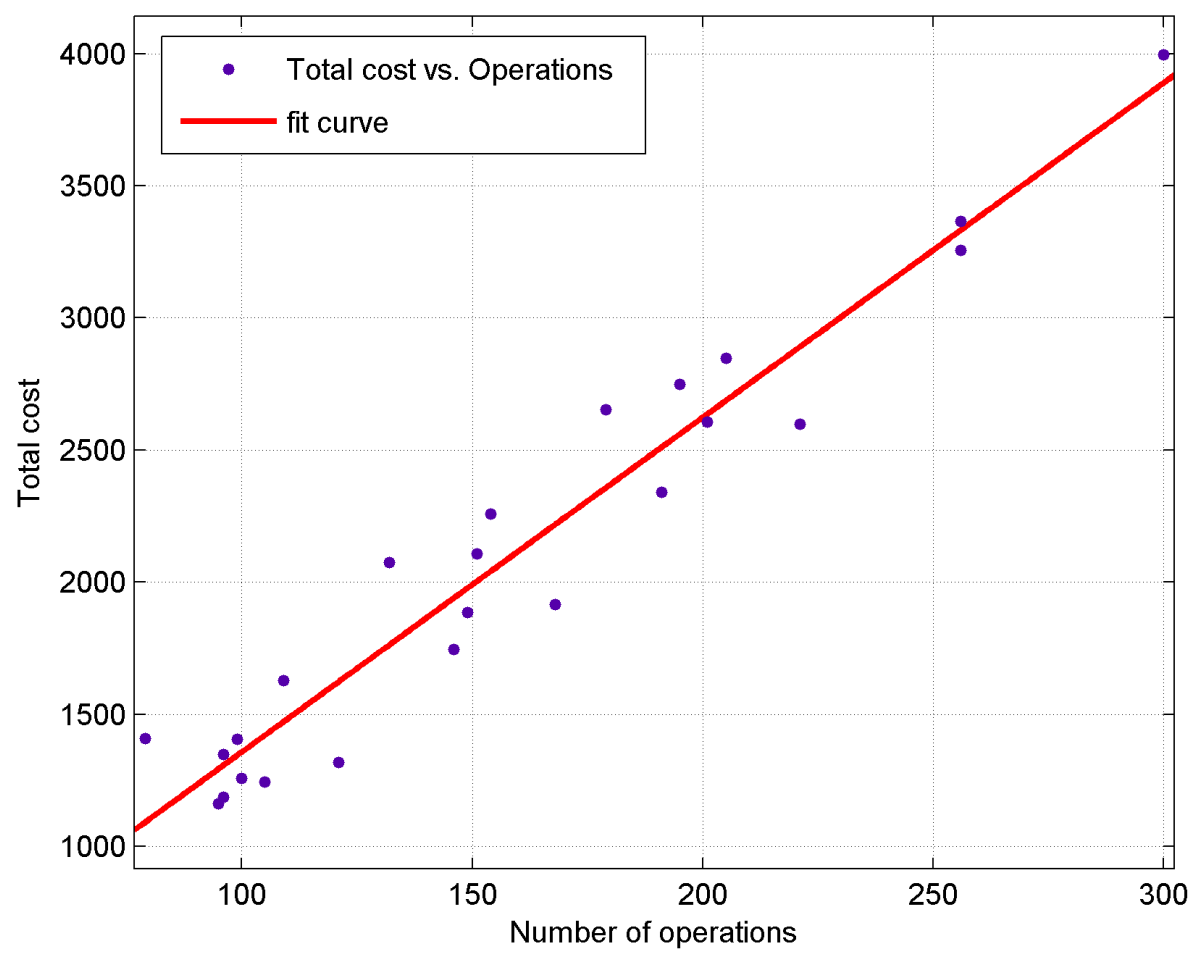

Figure 7. Relationship between total cost and the number of operations.

\section{Conclusions}

This paper mainly discusses the modeling and optimization method for network graph based process planning problems in the context of carbon emission reduction. In this research, we establish a novel mathematical model for process planning optimization where parts are expressed using a network graph. Operation precedence constraints have been considered and added to the proposed 
model to make the model more reasonable. Due to the complexity of the problem, we present a hybrid NSGA-II algorithm for both carbon emission and machining cost reduction. Based on the characteristics of the bi-objective optimization problem, a problem oriented local search method is developed and adopted in hybrid NSGA-II to explore a more competitive Pareto front in the solution space. In addition, the TOPSIS decision method is included in the algorithm to determine the ideal solution from the optimal non-dominated solutions.

Typical benchmark instances have been tested using the proposed algorithm. Experimental results indicate that the proposed hybrid NSGA-II is much better than the plain NSGA-II algorithm in solution quality because an effective local search method is employed in the algorithm. Meanwhile, both economic and environmental criteria can be achieved with compromise. The results also reveal that total costs and carbon emissions are not completely independent variables; both the total cost criterion and the carbon emission criterion are sensitive to the number of operations in process plans; and there is an approximately linear relationship between total carbon emission (as well as total cost) and the number of operations. A good suggestion is that one should choose process plans that contain less operations.

In this research, some parameters, such as tools and TADs, are not available in advance while some parameters, e.g., powers of 15 machines and the parameters of cutting tools, are either generated by the authors by sampling in a given interval or taken from existing literature. However, this will not hinder the application of the proposed method in the cases where real-world parameters are available.

This research is performed from a macro level. As a future research direction, more manufacturing details can be introduced within the network graph framework to optimize costs and carbon emission in process planning. Of course, the energy needed for transportation of the work pieces from one to another machine is also responsible for carbon emission in manufacturing sectors; this energy consumption is related to the layout of the factories or workshops and to the sequence of operations. Therefore, in further research, vehicle path planning as well as the carbon emission of automatic guided vehicles (AGVs) can be integrated into process planning problems.

Acknowledgments: This research is supported by the Funds for the National Natural Science Foundation of China (No. 51575211), International Cooperation and Exchange of the National Natural Science Foundation of China (No. 51561125002), Youth Natural Science Foundation of Zhejiang Province (No. LQ18E050006), the scientific research start-up project of Shaoxing University (No. 20165012), and the 111 Project of China (No. B16019).

Author Contributions: J. Huang and L. Jin conceived the experiments; J. Huang designed and performed the experiments; C. Zhang and L. Jin analyzed the data; and J. Huang wrote the paper.

Conflicts of Interest: The authors declare no conflict of interest.

\section{References}

1. Lv, S.; Qiao, L. A cross-entropy-based approach for the optimization of flexible process planning. Int. J. Adv. Manuf. Technol. 2013, 68, 2099-2110.

2. Liu, X.J.; Yi, H.; Ni, Z.H. Application of ant colony optimization algorithm in process planning optimization. J. Intell. Manuf. 2013, 24,1-13.

3. Lian, K.; Zhang, C.; Li, X.; Gao, L. An Effective Hybrid Genetic Simulated Annealing Algorithm for Process Planning Problem. In Proceedings of the 2009 Fifth International Conference on Natural Computation, Tianjin, China, 14-16 August 2009; Volume 5, pp. 367-373.

4. Lian, K.; Zhang, C.; Shao, X.; Gao, L. Optimization of process planning with various flexibilities using an imperialist competitive algorithm. Int. J. Adv. Manuf. Technol. 2012, 59, 815-828.

5. Shin, K.S.; Park, J.O.; Kim, Y.K. Multi-objective FMS process planning with various flexibilities using a symbiotic evolutionary algorithm. Comput. Oper. Res. 2011, 38, 702-712.

6. Guo, Y.W.; Mileham, A.R.; Owen, G.W.; Li, W.D. Operation sequencing optimization using a particle swarm optimization approach. Proc. Inst. Mech. Eng. Part B J. Eng. Manuf. 2006, 220, 1945-1958.

7. Salehi, M.; Tavakkoli-Moghaddam, R. Application of genetic algorithm to computer-aided process planning in preliminary and detailed planning. Eng. Appl. Artif. Intell. 2009, 22, 1179-1187. 
8. Dornfeld, D.A. Moving towards green and sustainable manufacturing. Int. J. Precis. Eng. Manuf. Green Technol. 2014, 1, 63-66.

9. Xu, J.; Wang, L. A Feedback Control Method for Addressing the Production Scheduling Problem by Considering Energy Consumption and Makespan. Sustainability 2017, 9, 1185.

10. Lu, C.; Gao, L.; Li, X.; Chen, P. Energy-efficient multi-pass turning operation using multi-objective backtracking search algorithm. J. Clean. Prod. 2016, 137, 1516-1531.

11. Zhao, F.; Murray, V.R.; Ramani, K.; Sutherland, J.W. Toward the development of process plans with reduced environmental impacts. Front. Mech. Eng. 2012, 7, 231-246.

12. Dai, M.; Tang, D.; Giret, A.; Salido, M.A.; Li, W. Energy-efficient scheduling for a flexible flow shop using an improved genetic-simulated annealing algorithm. Robot. Comput. Integr. Manuf. 2013, 29, 418-429.

13. Lin, W.; Yu, D.; Zhang, C.; Liu, X.; Zhang, S.; Tian, Y.; Liu, S.; Xie, Z. A multi-objective teaching-learning-based optimization algorithm to scheduling in turning processes for minimizing makespan and carbon footprint. J. Clean. Prod. 2015, 101, 337-347.

14. Yin, L.; Li, X.; Lu, C.; Gao, L. Energy-Efficient Scheduling Problem Using an Effective Hybrid Multi-Objective Evolutionary Algorithm. Sustainability 2016, 8, 1268.

15. Wang, F.; Rao, Y.; Zhang, C.; Tang, Q.; Zhang, L. Estimation of Distribution Algorithm for Energy-Efficient Scheduling in Turning Processes. Sustainability 2016, 8, 762.

16. Sun, Z.; Gu, X. Hybrid Algorithm Based on an Estimation of Distribution Algorithm and Cuckoo Search for the No Idle Permutation Flow Shop Scheduling Problem with the Total Tardiness Criterion Minimization. Sustainability 2017, 9, 953.

17. Wang, S.; Lu, X.; Li, X.; Li, W. A systematic approach of process planning and scheduling optimization for sustainable machining. J. Clean. Prod. 2015, 87, 914-929.

18. Yi, Q.; Li, C.; Zhang, X.; Liu, F.; Tang, Y. An optimization model of machining process route for low carbon manufacturing. Int. J. Adv. Manuf. Technol. 2015, 80, 1181-1196.

19. Ding, J.Y.; Song, S.; Wu, C. Carbon-efficient scheduling of flow shops by multi-objective optimization. Eur. J. Oper. Res. 2016, 248, 758-771.

20. Hassine, H.; Barkallah, M.; Bellacicco, A.; Louati, J.; Riviere, A.; Haddar, M. Multi objective optimization for sustainable manufacturing, application in turning. Int. J. Simul. Model. 2015, 14, 98-109.

21. Liu, Z.; Sun, D.; Lin, C.; Zhao, X.; Yang, Y. Multi-objective optimization of the operating conditions in a cutting process based on low carbon emission costs. J. Clean. Prod. 2016, 124, 266-275.

22. Shin, S.J.; Suh, S.H.; Stroud, I. A green productivity based process planning system for a machining process. Int. J. Prod. Res. 2015, 53, 5085-5105.

23. Li, X.; Li, W.; Cai, X.; He, F. A hybrid optimization approach for sustainable process planning and scheduling. Integr. Comput. Aided Eng. 2015, 22, 311-326.

24. Yin, R.; Cao, H.; Li, H.; Sutherland, J.W. A process planning method for reduced carbon emissions. Int. J. Comput. Integr. Manuf. 2014, 27, 1175-1186.

25. Kim, Y.K.; Park, K.; Ko, J. A symbiotic evolutionary algorithm for the integration of process planning and job shop scheduling. Comput. Oper. Res. 2003, 30, 1151-1171.

26. Zhang, L.; Wong, T. An object-coding genetic algorithm for integrated process planning and scheduling. Eur. J. Oper. Res. 2015, 244, 434-444.

27. Jin, L.; Tang, Q.; Zhang, C.; Shao, X.; Tian, G. More MILP models for integrated process planning and scheduling. Int. J. Prod. Res. 2016, 54, 4387-4402.

28. Li, C.; Tang, Y.; Cui, L.; Li, P. A quantitative approach to analyze carbon emissions of CNC-based machining systems. J. Intell. Manuf. 2015, 26, 911-922.

29. Bell, D.D.; Chou, J.; Nowag, L.; Liang, S.Y. Modeling of the Environmental Effect of Cutting Fluid. Tribol. Trans. 1999, 42, 168-173.

30. Dahmus, J.B.; Gutowski, T.G. An environmental analysis of machining. In Proceedings of the ASME International Mechanical Engineering Congress and RD\&D Exposition, Anaheim, CA, USA, 13-19 November 2004; pp. 13-19.

31. Li, W.D.; Ong, S.K.; Nee, A.Y.C. Hybrid genetic algorithm and simulated annealing approach for the optimization of process plans for prismatic parts. Int. J. Prod. Res. 2002, 40, 1899-1922. 
32. Rajemi, M.; Mativenga, P.; Aramcharoen, A. Sustainable machining: Selection of optimum turning conditions based on minimum energy considerations. J. Clean. Prod. 2010, 18, 1059-1065.

33. Tseng, H.E. Guided genetic algorithms for solving a larger constraint assembly problem. Int. J. Prod. Res. 2006, 44, 601-625.

34. Zhang, F.; Zhang, Y.F.; Nee, A.Y.C. Using genetic algorithms in process planning for job shop machining. IEEE Trans. Evol. Comput. 1997, 1, 278-289.

35. Deb, K.; Pratap, A.; Agarwal, S.; Meyarivan, T. A fast and elitist multiobjective genetic algorithm: NSGA-II. IEEE Trans. Evol. Comput. 2002, 6, 182-197.

(C) 2017 by the authors. Licensee MDPI, Basel, Switzerland. This article is an open access article distributed under the terms and conditions of the Creative Commons Attribution (CC BY) license (http:// creativecommons.org/licenses/by/4.0/). 